RADIOLOGY-TECHNICAL ARTICLE

\title{
Electrolytic echo enhancement: A novel method to make needles more reflective to ultrasound
}

\author{
John F. Cockburn ${ }^{1}$ and Stefan K. Khosh ${ }^{2}$ \\ ${ }^{1}$ Department of Imaging, The Canberra Hospital, Australian National University, Canberra, Australian Capital Territory, and ${ }^{2}$ Department of Imaging, \\ Austin Medical Centre, Melbourne, Victoria, Australia
}

\author{
JF Cockburn MRCP FRCR FFRRCSI; SK Khosh \\ FRANZCR.

\section{Correspondence \\ A/Professor John Cockburn, Department of Imaging, The Canberra Hospital, Yamba Drive, Garran, ACT 2605, Australia. \\ Email: john.cockburn@act.gov.au \\ Conflict of interest: None.}

Submitted 5 March 2013; accepted 2 July 2013.

doi:10.1111/1754-9485.12103

\begin{abstract}
Introduction: This study examines the effect of augmenting the ultrasound reflectivity of needles using a novel electrolytic echo enhancement method. Methods: Needles were connected by a lead to the negative terminal of a $4.5 \mathrm{~V}$ direct current source. A grounding pad, connected to the positive terminal, was positioned on the undersurface of an ex vivo ox liver phantom. During needle insertion into the liver, electrolysis was induced creating a layer of gas on the needle electrode.

Results: Analysis of images showed a significant increase in needle brightness using electrolytic echo enhancement. Brightness was found to increase by a factor of $\times 3.6$ compared with controls $(P<0.001)$.

Conclusion: Electrolytic echo enhancement has the potential to make ultrasound-guided procedures safer and quicker for patients and increase the confidence of operators in their ability to see the whole needle including its tip.
\end{abstract}

Key words: electrolytic; enhancement; needle; reflectivity; ultrasound.

\section{Introduction}

Ultrasound guidance is central to many percutaneous interventional procedures performed today. The readily available, inexpensive and flexible nature of ultrasound, combined with its excellent safety profile, is responsible for the dramatic increase in ultrasound-guided procedures over the past three decades. Ultrasound was first used to guide procedures using an improvised static scanner. ${ }^{1}$ Subsequently, various methods and instruments were devised to improve the ability to see biopsy needles and catheters using ultrasound, ${ }^{2-4}$ relying primarily on a combination of skilled technique and mechanically establishing a fixed angle between the ultrasound beam and the needle trajectory. Improvements in the design and performance of ultrasound machines and needle-biopsy guides, ${ }^{5-8}$ various augmentation techniques, ${ }^{9-19}$ and new computer algorithms and material technology ${ }^{20-27}$ have led to even more enhanced visualisation methods. Despite these advances, problems with needle visualisation remain. ${ }^{28,29}$

In this paper, we describe a simple electrolytic circuit which augments needle reflectivity by capitalising on the extreme hyperreflectivity of gas to ultrasound. We present the initial results of a series of experiments using electrolytic echo enhancement (ELEE).

\section{Methods}

A $15-\mathrm{cm}$ long 22G Chiba biopsy needle (Cook Medical, Bloomington, IN, USA) was connected by conducting wire to the negative terminal of a variable direct current source (Digitor direct current adapter, Dick Smith electronics, Sydney, NSW, Australia). A standard electrocardiogram (ECG) 'dot' was placed against a large ex vivo piece of ox liver in a plastic container and connected by conducting wire to the positive terminal of the adapter. When the needle is rendered negative (cathodic) with respect to the reference electrode, hydrogen gas is produced. A liver tissue medium was chosen owing to its relative hyperreflectivity compared with liquid and artificial phantoms. This feature was felt more likely to simulate the problems faced by radiologists during ultrasound-guided procedures in patients. The needle was connected to the negative terminal of the direct current (DC) source, set at $4.5 \mathrm{~V}$, and was then 

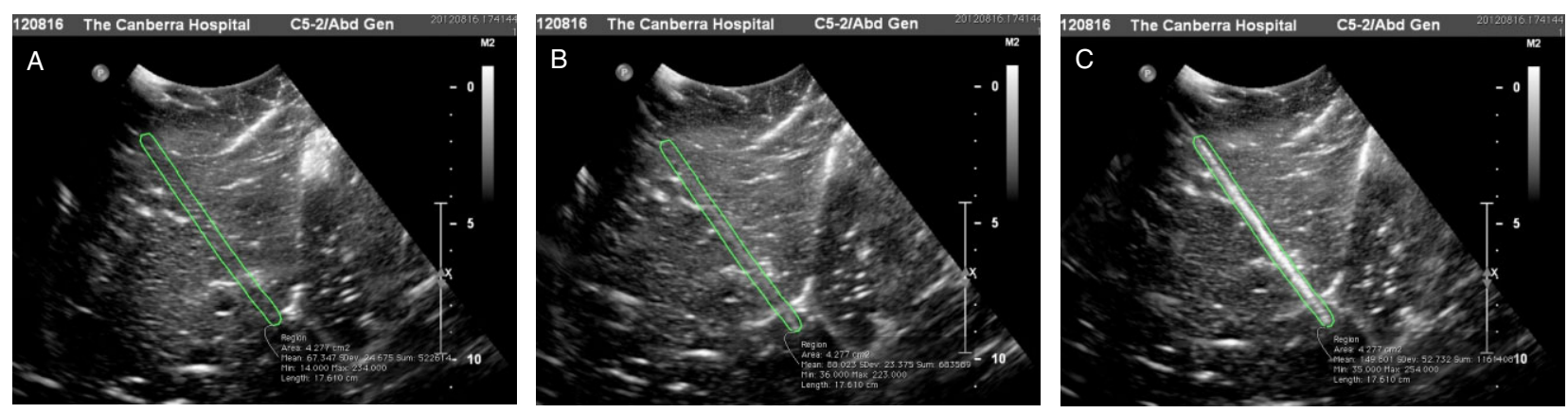

Fig. 1. Background region of interest drawn around ex vivo liver gives a mean brightness value of 67.347 (a). Following insertion of a $22 \mathrm{G}$ Chiba needle control, the brightness of the region of interest increases to a mean of 88.023 (b). The electrolytically echo-enhanced needle increases the brightness in the region of interest to a mean value of 149.801 (c)

introduced at an angle of $45^{\circ}$ to a depth of between 5 and $10 \mathrm{~cm}$. Confirmation of the voltage was achieved using a multi-meter which measured the potential between the needle and the ECG dot. Needle insertion was monitored using the $5-2 \mathrm{MHz}$ curved array probe of a diagnostic ultrasound machine (Philips Ultrasound iU22, Philips, Bothell, WA, USA). Pilot studies of various voltages between 0 and $12 \mathrm{~V}$ were performed to choose a voltage and duration of voltage application so that needle brightness appeared to be improved during insertion. Based on these observations, $4.5 \mathrm{~V}$ was chosen to be applied for $6 \mathrm{~s}$. The time taken between the start and end of needle insertion was subsequently limited to $6 \mathrm{~s}$ to standardise the amount of electrical energy delivered. Three images were taken for each insertion series: an initial static image of the background liver prior to insertion, an image of an inserted control needle disconnected from DC and an image of an inserted needle connected to the active DC source. Based on previous observations, 11 series were performed in total in order to achieve statistical significance. Twelve new needles were used: a single control and 11 electrolytically enhanced needles. None of the ultrasound parameters were changed from the start of the experiment until all 33 insertions had been completed. Each image was transferred to a computer running OSIRIXIMAGE manipulation software (Pixmeo SARL, Bernex, Switzerland). An identical region of interest, created using copy and paste, was drawn around each of the three images, and a mean grayscale measurement was derived. The data were analysed using a statistics software programme (SPSS version 19, IBM, Chicago, IL, USA) running Student's paired $t$-test. This package creates means, standard deviations (SDs), 95\% confidence intervals and two-tailed $P$ values. Comparison was made between background, control and ELEE to assess the degree to which the brightness of the control and electrolytic needles appeared with respect to the background. Each series of three insertions was repeated 11 times. An approximation of the amount of gas produced by electrolysis during needle insertion was calculated using a combination of Faraday's first law of electrolysis and the ideal gas equation.

\section{Results}

The ultrasound images were obtained in sets of three: a background image (Fig. 1a), an image following insertion of a control needle (Fig. 1b) and an image following insertion of an ELEE needle (Fig. 1c).

The brightness values were calculated by the OSIRIXIMAGE software (Table 1) and exported to the SPSS programme for statistical analysis.

The collected data distributions of control and electrolytic needles met the assumptions of normality. The mean background grayscale measurement prior to needle insertion was 82.246 units $(S D=8.373)$. The mean measurement following insertion of a control needle was 97.297 units $(S D=8.084)$. The mean measurement following insertion of an ELEE needle was 136.57 units $(S D=8.692)$. Comparison of the mean percent change from background (control 18.78\% $(\mathrm{SD}=8.71$, standard error of the mean $(\mathrm{SEM})=2.62)$

Table 1. Measurements of brightness within regions of interest for the 11 repeated needle insertion series

\begin{tabular}{lccc}
\hline & Background & Control needle & ELEE needle \\
\hline 1 & 93.5 & 99.7 & 146.94 \\
2 & 96.004 & 101.69 & 132.95 \\
3 & 82.676 & 101.91 & 128.61 \\
4 & 67.347 & 88.023 & 149.8 \\
5 & 86.079 & 99.871 & 139.62 \\
6 & 77.852 & 99.402 & 140.9 \\
7 & 74.524 & 84.646 & 127.26 \\
8 & 87.956 & 108.74 & 146.27 \\
9 & 82.808 & 107.22 & 135.27 \\
10 & 77.942 & 89.452 & 130.06 \\
11 & 78.023 & 89.609 & 124.59 \\
\hline
\end{tabular}

ELEE, electrolytic echo enhancement. 
Table 2. Statistical analysis of brightness

\begin{tabular}{lc}
\hline Measure & Mean $(95 \% \mathrm{Cl})$ \\
\hline Background & $82.246(76.62,87.87) \mathrm{SD}=8.37, \mathrm{SEM}=2.52$ \\
Control needle & $97.297(91.87,102.73) \mathrm{SD}=8.08, \mathrm{SEM}=2.44$ \\
ELEE & $136.57(130.73,142.41) \mathrm{SD}=8.69, \mathrm{SEM}=2.62$ \\
\hline Mean percent difference from background $(95 \% \mathrm{Cl})$ \\
\hline Control & $18.78 \%(12.93,24.63) \mathrm{SD}=8.71, \mathrm{SEM}=2.63$ \\
ELEE & $67.62 \%(53.51,81.72) \mathrm{SD}=20.99, \mathrm{SEM}=6.33$ \\
\hline
\end{tabular}

$\mathrm{Cl}$, confidence interval; SD, standard deviation; SEM, standard error of the mean; ELEE, electrolytic echo enhancement.

and ELEE $67.62 \%($ SD $=20.99$, SEM $=6.33)$ ) showed that application of a $4.5 \mathrm{~V}$ current over a $6 \mathrm{~s}$ period increased the brightness of the region of interest of the electrolytic needle by a factor of $\times 3.6$ compared with the control ( $T-13.310$ (d.f. 10), $P<0.001$ ) (Table 2).

The amount of gas produced by a needle at $4.5 \mathrm{~V}$ was calculated to be approximately 4 thousandths of a millilitre per second during insertion.

\section{Discussion}

Our initial experiments demonstrate the effect of using $4.5 \mathrm{~V}$ of direct current to turn a needle within tissue into a negatively charged electrode which, along with a positively charged grounding pad, establishes an electrical circuit within a block of tissue. This electrical energy has voltage sufficient to split the saline environment of tissue according to a simple electrolysis equation: $2 \mathrm{NaCL}+2 \mathrm{H}_{2} \mathrm{O} \rightarrow \mathrm{H}_{2}+\mathrm{Cl}_{2}+2 \mathrm{NaOH}$. As a result, hydrogen gas is liberated from the negatively charged cathode and chlorine from the positively charged anode. The hydrogen forms on the needle cathode and renders it hyperechoic - appearing extremely bright on diagnostic ultrasound images. A tiny amount of chlorine gas is formed at the grounding pad over a relatively much wider area compared with the needle shaft.

Using $4.5 \mathrm{~V}$, it takes less than $6 \mathrm{~s}$ for the needle to subjectively become progressively very hyperreflective. This increase in reflectivity can be observed as soon as the circuit is made and gas begins to form (i.e. as soon as the needle penetrates the liver surface). When the needle is rendered negative (cathodic) with respect to the reference electrode, hydrogen gas is produced. When polarity is reversed, chlorine gas is produced. For the purpose of this series of experiments, a negatively charged needle was chosen. The effect of different gas production is the subject of further experimentation. A $4.5 \mathrm{~V}$ was used in this experiment because in pilot studies, we have found that lower voltages, while also making needles hyperechoic, can take substantially longer than $6 \mathrm{~s}$ : the lower the voltage, the longer it takes sufficient gas to form on the needle to influence reflec- tivity. Higher voltages, such as $9 \mathrm{~V}$, create a brighter needle instantly; however, excess gas production over a period of seconds can lead to an undesirable widening of the needle appearance on the image. We would anticipate in a clinical situation that the sensation of $9 \mathrm{~V}$ in tissue could be unpleasant (analogous to the sensation when a $9 \mathrm{~V}$ transistor battery is applied to the tongue). A $22 \mathrm{G}$ needle was used, but we have observed in other experiments that the effect of increased reflectivity applies to all of the common needle sizes. We chose $45^{\circ}$ insertions because we felt that this is relatively representative of typical procedures. We have observed that the hyperreflectivity appears to be largely independent of the angle of insertion owing to the intense backscatter from the bubbles. We further observed that the entire needle shaft and tip were rendered hyperreflective and that the needle tip was readily identifiable. Images of our control needle insertions showed slightly variable reflectivity (Fig. 1b). We ascribe this variability to the physics of the needle tip echo, which tends to be brighter than that arising from the shaft, ${ }^{15}$ to slight bending of needles during insertion, and to the effect of the curved array probe. Similar inhomogeneity was occasionally observed during ELEE needle insertions (Fig. 1c). By moving the ultrasound probe slightly, we found that it was possible to demonstrate homogeneous-enhanced reflectivity along the entire needle shaft.

The safety of creating gas within tissue using direct current has been studied in the field of tissue ablation in large animal models and in man. Such ablation experiments use a far greater amount of electrical energy without significant reported adverse events. ${ }^{30-32} \mathrm{~A}$ further example of the safety of direct current in vivo occurs during neuro-intervention when electrolysis is used to detach coils when treating intracranial aneurysms. Electrolytic detachable coil devices use between $12 \mathrm{~V}$ and $28 \mathrm{~V}$ depending on the type of coil and a considerably higher amount of electrical energy ${ }^{33}$ and therefore gas formation.

During the formation of hydrogen bubbles on the needle, the same number of moles of chlorine gas is released at the ECG dot over a wider surface area. We have found that this did not cause any visible change on the liver phantom surface, but there are theoretical issues which might arise if electrolysis was used in patients in this way. Once again, however, experiments using direct current to ablate liver tumours in patients have shown that the much larger DC currents involved do not cause significant tissue damage at the reference electrode site. ${ }^{32}$

From an imaging standpoint, an interesting feature of electrolytic needle insertion is that a line of hydrogen bubbles is retained within the tissue phantom following withdrawal of the needle. It is evident that the bubbles can be 'rubbed off' during needle insertion and withdrawal. We have found that, when many passes are made, lines of retained bubbles can interfere with 
the identification of the needle tip. This perceptual problem can be eliminated, however, by moving the needle slightly: only the line of 'true' needle bubbles moves.

A theoretical limitation of this study was the subjectivity involved in choosing images showing the longest brightest path which included the needle tip. Further error was introduced when a region of interest was drawn around the electrolytic needle rather than placing a fixed parallelogram region of interest. This region of interest was made sufficiently wide to accommodate both the control and electrolytic needle insertions. The freehand method was chosen in order to make allowances for any bending of needles which can occur and the slight distortion caused by the curved array probe. Measuring the brightness of the background (using one region of interest copied and pasted onto the background) goes some way towards mitigating errors caused by our methodology. We found that tiny amounts of room air adherent to the needle tip are introduced during all control and electrolytic needle insertions. These cause slight differences in the region of interest during each needle insertion. A further possible confounding effect is change in the electrochemical composition of ex vivo liver owing to repeated tissue electrolysis. Assessment of the magnitude of these limitations is difficult; however, we believe the latter to be of no consequence, as the amount of gas produced by the needle, measured as image brightness, did not vary from the start to the end of the experiment.

Over the last couple of decades, advances in diagnostic ultrasound machine technology as well as needle manufacture have made needles easier to see using ultrasound. Despite this, there is an ongoing demand for further improvements which render needles more visible.

\section{Conclusion}

In this paper, we describe a new simple electrolytic circuit which augments needle reflectivity by capitalising on the extreme hyperreflectivity of gas to ultrasound and present a series of experiments using ELEE. Our results suggest that electrolysis has the exciting potential to make conductive apparatus, including needles, substantially more visible to ultrasound.

\section{References}

1. Holm HH, Kristensen JK, Rasmussen SN, Northeved A, Barlebo $\mathrm{H}$. Ultrasound as a guide in percutaneous puncture technique. Ultrasonics 1972; 10: 83-6.

2. Yeh EL. An ultrasonic deflector for aspiration and biopsy. J Clin Ultrasound 1975; 3: 125-7.

3. Yeh HC, Mitty HA, Wolf BS. A simple ultrasound guide for needle puncture. J Clin Ultrasound 1976; 4: $53-4$.
4. Pedersen JF. Percutaneous puncture guided by ultrasonic multitransducer scanning. J Clin Ultrasound 1977; 5: 175-7.

5. Goldberg BB, Cole-Beuglet C, Kurtz AB, Rubin CS. Real-time aspiration-biopsy transducer. J Clin Ultrasound 1980; 8: 107-12.

6. Buonocore E, Skipper GJ. Steerable real-time sonographically guided needle biopsy. AJR Am J Roentgenol 1981; 136: 387-92.

7. Heckemann R, Seidel KJ. [In vitro and in vivo demonstrations of puncture instruments in the sonographic real time image. 1: puncture needles]. Ultraschall Med 1982; 3: 18-23.

8. Reid MH. Real-time sonographic needle biopsy guide. AJR Am J Roentgenol 1983; 140: 162-3.

9. Lee TG, Knochel JQ. Air as an ultrasound contrast marker for accurate determination of needle placement. Tumor biopsy localization and other applications. Radiology 1982; 143: 787-8.

10. Reading CC, Charboneau JW, Felmlee JP, James EM. US-guided percutaneous biopsy: use of a screw biopsy stylet to aid needle detection. Radiology 1987; 163: 280-1.

11. Kurohiji T, Sigel B, Justin J, Machi J. Motion marking in color Doppler ultrasound needle and catheter visualization. J Ultrasound Med 1990; 9: 243-5.

12. Bisceglia M, Matalon TA, Silver B. The pump maneuver: an atraumatic adjunct to enhance US needle tip localization. Radiology 1990; 176: 867-8.

13. Winsberg F, Mitty HA, Shapiro RS, Yeh HC. Use of an acoustic transponder for US visualization of biopsy needles. Radiology 1991; 180: 877-8.

14. Perrella RR, Kimme-Smith C, Tessler FN, Ragavendra N, Grant EG. A new electronically enhanced biopsy system: value in improving needle-tip visibility during sonographically guided interventional procedures. AJR Am J Roentgenol 1992; 158: 195-8.

15. Bondestam S. The needle tip echo. J Ultrasound Med 1992; 11: 253-6.

16. Laine HR, Rainio J. An inexpensive method of improving visualisation of the needle tip in fine needle aspiration biopsy (FNAB). Ann Chir Gynaecol 1993; 82: 43-5.

17. Cockburn JF, Cosgrove DO. Device to enhance visibility of needle or catheter tip at color Doppler US. Radiology 1995; 195: 570-2.

18. Gottlieb RH, Robinette WB, Rubens DJ, Hartley DF, Fultz PJ, Violante MR. Coating agent permits improved visualization of biopsy needles during sonography. AJR Am J Roentgenol 1998; 171: 1301-2.

19. Feld R, Needleman L, Goldberg BB. Use of needle-vibrating device and color Doppler imaging for sonographically guided invasive procedures. AJR Am J Roentgenol 1997; 168: 255-6.

20. Draper KJ, Blake CC, Gowman L, Downey DB, Fenster A. An algorithm for automatic needle localization in ultrasound-guided breast biopsies. Med Phys 2000; 27: 1971-9. 
21. Howard MH, Nelson RC, Paulson EK, Kliewer MA, Sheafor $\mathrm{DH}$. An electronic device for needle placement during sonographically guided percutaneous intervention. Radiology 2001; 218: 905-11.

22. Saleh A, Ernst S, Grust A, Fürst G, Dall P, Mödder U. [Real-time compound imaging: improved visibility of puncture needles and localization wires as compared to single-line ultrasonography]. Rofo 2001; 173: 368-72.

23. Sauer F, Khamene A, Bascle B, Vogt S. An augmented reality system for ultrasound guided needle biopsies. Stud Health Technol Inform 2002; 85: 455-60.

24. Li T, Gianchandani RY, Gianchandani YB. Micromachined bulk PZT tissue contrast sensor for fine needle aspiration biopsy. Lab Chip 2007; 7: 179-85.

25. Tsui $\mathbf{B C H}$. Facilitating needle alignment in-plane to an ultrasound beam using a portable laser unit. Reg Anesth Pain Med 2007; 32: 84-8.

26. Bluvol N, Sheikh A, Kornecki A, Fernandez DDR, Downey $D$, Fenster A. A needle guidance system for biopsy and therapy using two-dimensional ultrasound. Med Phys 2008; 35: 617-28.

27. Takayama W, Yasumura R, Kaneko T et al. [Novel echogenic needle for ultrasound-guided peripheral nerve block 'Hakko type CCR']. Masui 2009; 58: 503-7.
28. Blaivas M, Adhikari S. An unseen danger: frequency of posterior vessel wall penetration by needles during attempts to place internal jugular vein central catheters using ultrasound guidance. Crit Care Med 2009; 37: 2345-9.

29. Moak JH, Lyons MS, Wright SW, Lindsell CJ. Needle and guidewire visualization in ultrasound-guided internal jugular vein cannulation. Am J Emerg Med 2011; 29: 432-6.

30. Dobbins C, Brennan C, Wemyss-Holden S, Cockburn J, Maddern G. Bimodal electric tissue ablation - long term studies of morbidity and pathological change. J Surg Res 2008; 148: 251-9.

31. Robertson GS, Wemyss-Holden SA, Dennison AR, Hall PM, Baxter $\mathrm{P}$, Maddern GJ. Experimental study of electrolysis-induced hepatic necrosis. Br J Surg 1998; 85: $1212-6$.

32. Wemyss-Holden SA, Berry DP, Robertson GS, Dennison AR, De La M Hall P, Maddern GJ.

Electrolytic ablation as an adjunct to liver resection: safety and efficacy in patients. ANZ J Surg 2002; 72: 589-93.

33. InZone e-Detachment System (Boston Scientific Corporation, Natick, MA, USA). United States FDA $510(k)$ Summary of Safety and Effectiveness, clearance under pre-market notification K02 1494, June 2002. 